\title{
ARTICLE
}

\section{Psychiatric assessment and treatment of survivors of torture}

\author{
Richard M. Duffy \& Brendan D. Kelly
}

Richard M. Duffy is a special lecturer at University College Dublin and a senior registrar in general adult psychiatry at the Mater Misericordiae University Hospital, Dublin. He is also a researcher with Spiritan Asylum Services Initiative (SPIRASI) in Dublin. SPIRASI is a humanitarian non-governmental organisation that works with asylum seekers, refugees and other disadvantaged migrant groups, with special concern for survivors of torture. It is the only centre in Ireland for the treatment of survivors of torture.

Brendan D. Kelly is Associate Clinical Professor of Psychiatry at University College Dublin and a consultant psychiatrist at the Mater Misericordiae University Hospital, Dublin. He is a member of the board of SPIRASI, and in addition to his medical degree, he holds doctorates in medicine (MD), history (PhD), governance (DGov) and law (PhD). He has published over 200 peer-reviewed papers. Correspondence Dr Richard M. Duffy, Department of Adult Psychiatry, School of Medicine and Medical Science, University College Dublin, Dublin, Ireland 2. Email: richard.duffy@ucd.ie

\begin{abstract}
SUMMARY
Torture remains an ongoing global problem. Many individuals who have migrated from areas of conflict and political instability have suffered some form of torture. Survivors of torture can present significant challenges to general practitioners, voluntary bodies and mental health services. This article describes the many physical, psychological and social effects of torture. An understanding of these will hopefully aid comprehensive assessment of survivors of torture by local mental health teams. Also described are the current treatment options and their evidence base.
\end{abstract}

\section{LEARNING OBJECTIVES}

- Appreciate the prevalence and current epidemiological trends of torture and its relation to migration

- Be aware of all important components of a comprehensive psychiatric assessment of survivors of torture

- Understand the evidence-based treatments and their theory

\section{DECLARATION OF INTEREST}

None

Torture (Box 1) has been pervasive throughout human history. It has been used by governments, sanctioned by kings, perpetrated by religious organisations and performed by countless individuals. Motivation behind it has ranged from vengeance to religious observance and from information extraction to judicial punishment. Torture is condemned in article 3 of the European Convention on Human Rights and its implications to psychiatry have been discussed in this journal (Curtice 2010).

Despite many steps taken to eradicate it, torture is still thought to be practised in over 130 countries (Wenzel 2007; International Rehabilitation Council for Torture Victims 2012). Amnesty International reports that in 2009, 79\% of G-20 countries were practising torture or otherwise illtreating individuals (Amnesty International 2010). So this problem is not confined to low- and middleincome countries.
Epidemiological data on the prevalence of torture are inconsistent. Acquiring precise data on torture is complicated by the heterogeneity of populations and reluctance of these populations to engage with services, especially in a formal or clinical setting. Cultural differences, stigma and insecure residency status may all further hamper research attempts (Wenzel 2007). The lack of a consistent, universally accepted definition of torture (the United Nation's definition appears in Box 1) has resulted in conflicting and inconsistent findings in the area. If an excessively broad definition is used, the severity of the trauma suffered by survivors of torture can be minimised, preventing them from accessing services that they need.

Many victims of torture become refugees, which presents significant challenges for research because of the transitory nature of this population (Jaranson 2011). There are large geographical variations in the prevalence of torture among asylum seekers: as Masmas et al (2008) note: 'The prevalence of torture survivors seeking asylum will always reflect the existing national and international political situation'. Many physicians fail to enquire about experiences of torture, further reducing the known prevalence (Eisenman 2003).

B0X 1 What is torture?

Article 1 of the United Nation's Convention against Torture and Other Cruel, Inhuman or Degrading Treatment or Punishment defines torture as:

'any act by which severe pain or suffering, whether physical or mental, is intentionally inflicted on a person for such purposes as obtaining from him or a third person information or a confession, punishing him for an act he or a third person has committed or is suspected of having committed, or intimidating or coercing him or a third person, or for any reason based on discrimination of any kind, when such pain or suffering is inflicted by or at the instigation of or with the consent or acquiescence of a public official or other person acting in an official capacity.'

(United Nations 1984) 
The International Rehabilitation Council for Torture Victims (IRCT) supports the rehabilitation of victims of torture and works to prevent torture worldwide. It is an umbrella organisation for over 140 independent organisations working in over 70 countries. The IRCT estimates that its organisations treat over 100000 survivors of torture each year (IRCT 2012). Worldwide, there are more than 230 treatment programmes for survivors of torture (Jaranson 2011). These programmes are highly varied, depending on the location, available resources and the cultural context (IRCT 2010). They strive to provide long-term holistic rehabilitation treatments tailored to the social, economic, cultural and political setting.

\section{Torture among migrants}

Torture disproportionally affects marginalised groups (IRCT 2012). Such groups were identified in a study of Somali and Oromo women (Robertson 2006): older age, low educational attainment, poor fluency in English and increased family responsibilities were shown to be associated with increased prevalence of being subjected to torture.

A high proportion of forced migrants and asylum seekers have experienced torture. In a Danish study of asylum seekers carried out in 2008, $61 \%$ reported experiencing persecution and $45 \%$ reported that they had been exposed to torture (Masmas 2008); of those who had undergone torture, $31 \%$ had experienced it in the year before arriving in Denmark. A similar prevalence of torture among asylum seekers has been reported in other studies in Europe. For example, in another Danish study half of asylum seekers reported having been tortured (Montgomery 1994), and in a Norwegian study $57.5 \%$ reported such experiences (Jakobsen 2007). In US studies of foreign nationals, $6-11 \%$ had personal experience of torture (Eisenman 2003).

\section{Assessment of survivors of torture}

A comprehensive assessment of survivors of torture can only be performed with an in-depth knowledge of the consequences of torture. Its effects are varied and complex (Box 2). In addition to biological and psychological effects, there are social and cultural consequences (Jaranson 2011). None of the consequences occurs in isolation. Each affected domain influences other domains and exacerbates and modifies other symptoms. It is important to enquire about each of these domains during any assessment.

\section{The biological effects of torture}

Assistance from mental health services is often needed to ensure that survivors of torture gain access to the medical services they need. Consequently, assessment of their physical complaints is vital, and close liaison with general practitioners (GPs) and other doctors is key. In many ways, the physical effects of torture are the most obvious. There are both direct and indirect physical consequences of torture (Jaranson 2011).

The direct physical consequences result from injuries intentionally inflicted by the perpetrators of torture. The most common direct consequence is pain. The type and location of pain depends on the type of torture experienced. For example, victims of falanga torture (caning or beating of the soles of the feet) suffer chronic heel pain, altered sensation and abnormal gait. Individuals who have been suspended by their arms experience brachial plexus injuries, neurogenic pain, stretch arthritis syndrome, and impaired neurological sensations.

The indirect physical consequences of torture are physical problems experienced secondary to torture but not due to the physical injuries sustained. Indirect physical effects are no less serious and can be more debilitating than direct effects. They can include hypertension and chronic headaches. Individuals subjected to sexual trauma may also have acquired infections secondary to this or may experience fertility problems or sexual dysfunction. Survivors of torture have also been noted to have higher levels of respiratory problems (Shrestha 1998).

\section{B0X 2 Sequelae of torture}

\begin{tabular}{ll}
\hline Biological & Psychological \\
- Direct & - Anxiety \\
- Fractures & - Post-traumatic stress disorder (including \\
- Scars & possible complex PTSD) \\
- Acquired sexually transmitted infections & - Depression \\
- Frontal lobe damage & - Somatoform pain disorder \\
- Cognitive impairment from head trauma & - Substance misuse \\
- Malnutrition & Social \\
- Indirect & - Loss of social functioning \\
- Exacerbation of pre-existing medical & - Loss of status \\
condition & - Separation from friends and family \\
- Hypertension and cardiovascular & - Loss of social supports \\
disease & - Reduced quality of life \\
- Respiratory complaints & - Future uncertainty \\
- Chronic headaches & - Forced migration \\
- Sexual dysfunction &
\end{tabular}


Organic brain damage

Assessment by psychiatric services may be particularly important in the case of organic brain damage. Blunt force trauma, shaking, asphyxia and penetrating head injuries can all result in organic brain damage. Symptoms may include seizures, cognitive impairment, vertigo and headaches. These symptoms can be caused by a range of neurological conditions, including concussion syndrome, epilepsy and intracranial bleeding (Moreno 2002). Many survivors of torture report experiencing loss of consciousness, which is associated with post-traumatic seizures (Moreno 2002). The longer that somebody is unconsciousness, the higher the risk of future seizures. Temporal and frontal lobe seizures and frontal lobe damage can be particularly hard to diagnose, and diagnosis can be further complicated if the individual is outside of their culture of origin or does not speak the language of their host country. Multiple assessments or input from a neuropsychologist may aid assessment in such cases.

\section{The psychological effects of torture}

A full psychiatric history and mental state examination must be performed on survivors of torture presenting to mental health services. There is very strong evidence that torture and political violence lead to mental health problems (Eisenman 2003). A large meta-analysis (Steel 2009) revealed that some studies have shown a significantly more psychological problems in tortured than in nontortured populations of asylum seekers (Masmas 2008), whereas other studies have found similar psychological and physical health problems in both groups. This heterogeneity was also observed in a systematic review of mental disorder in refugees resettled in high-income countries (Fazel 2005), where different studies showed widely varying rates of major mental illness. Fazel et al did conclude, however, that these refugees were approximately 10 times more likely than the general population to suffer from post-traumatic stress disorder (PTSD). It is estimated that about $30 \%$ of refugees have PTSD (Steel 2009). These mental health problems are long lasting, with significant symptoms still present at 10 years (Carlsson 2006). Anxiety, PTSD and depression are all important to enquire about, but it is also important to acknowledge that many survivors of torture will experience mental health problems through somatic symptoms. Many individuals try to deal with their trauma through substance misuse, so screening for this is also essential.

\section{Anxiety}

High levels of anxiety are often seen during the assessment of individuals who have experienced torture. Of all mental health problems, anxiety has the strongest association with torture. There is a strong association between experiencing political violence and symptoms of panic disorder (Eisenman 2003). After correction for multiple potential confounding variables, symptoms of panic disorder were nearly five times as common in individuals exposed to political violence than in non-exposed individuals. Anxiety may relate to many things, including the individual's traumatic experience, their current living circumstances and their separation from friends and family.

\section{PTSD}

Symptoms of PTSD should always be enquired about. Individuals may have experienced additional trauma since their torture, especially if they have been navigating the asylum process. Steel et al (2009) note that PTSD is the most researched consequence of torture. Their metaanalysis of studies involving of over 80000 refugees and individuals who had lived in conflict zones in over 40 countries found that reporting torture had the strongest association with developing PTSD. Experiences of torture doubled the individual's risk of developing symptoms of PTSD. The usefulness of information from such a large metaanalysis must be questioned, as populations are as diverse as the social and political environments from which they come. However, in addition to the association, a dose-response relationship between torture and PTSD symptoms has been described, helping to establish causation (Johnson 2008). In a study of Latino immigrants living in the USA, higher levels of PTSD symptoms were found in a population who had been exposed to political violence compared with those who had no exposure (Eisenman 2003). Only 8\% of this population were survivors of torture, but it is fair to assume that the majority of survivors of torture have suffered political violence. This study also highlights the psychological consequences of political violence even in the absence of torture, which in itself can result in symptoms of PTSD.

\section{Complex PTSD}

In addition to what is traditionally meant by PTSD, there is a growing literature on 'complex PTSD' (McDonnell 2013) (Box 3). Although complex PTSD is not recognised in DSM-5, it has been proposed (Cloitre 2013) that it be included in ICD-11. Complex PTSD has been associated with 
BOX 3 Is complex PTSD a useful diagnosis?

Pros

- Growing biological basis

- Preliminary evidence supporting exposure-based treatments targeting dissociation

Cons

- Questionable current evidence supporting this as a discrete diagnostic category

- Not recognised in ICD-10 or DSM-5

- Limited current research on treatments and prognosis

repeated and sustained interpersonal trauma, so it has been suggested that this disorder may be common in survivors of torture. Complex PTSD differs from PTSD by also including dissociative symptoms, traits of emotional unstable personality disorder and somatisation symptoms. Recent research has demonstrated the clinical validity of these different diagnostic categories (Knefel 2013; Elklit 2014). In addition to clinical grounds for the diagnosis of complex PTSD, individuals experiencing dissociative symptoms in PTSD have been found to have differences on functional magnetic resonance imaging of the brain (Lanius 2012). These studies may address some of the diagnostic concerns that had been raised about complex PTSD (Resick 2012). This is a very important diagnosis to bear in mind during assessment, particularly in individuals who display emotionally unstable traits.

\section{Depression}

Steel et al's meta-analysis also highlighted the need to assess individuals for depression, as it found a significant relationship between reported torture and developing symptoms of depression (Steel 2009). Among displaced persons, the odds ratio for developing depression was 1.48 in those who had been tortured compared with those who had not been tortured, i.e. depression was almost 50\% more common among those who had experienced torture. This association was not as strong as the relationship between torture and PTSD. In Eisenman et al's study discussed above, political violence was also associated with increased depressive symptoms, even after controlling for multiple confounders (Eisenman 2003).

\section{Somatoform pain}

The artificial nature of the boundary between physical and psychological effects of torture is highlighted by somatoform disorders. Surveys of refugees with high proportions of torture survivors have shown high levels of somatoform pain disorders (van Ommeren 2004). Whatever their cause, physical or psychological, the symptoms of pain are long lasting: one study reported that all participants seen at a 10-year follow-up were still experiencing pain (Olsen 2007).

Pain may be the central focus of an assessment, as some individuals will be more comfortable discussing physical rather than psychological problems. Close interaction with other medical services is often needed to rule out medical conditions. Psychiatrists should be cautious of referrals for somatoform symptoms without comprehensive prior investigations.

\section{Substance misuse}

Screening for substance misuse is necessary in all assessments. Evidence for the prevalence of substance misuse among survivors of torture is limited and mixed. A study of Latino migrants living in the USA compared those who had experienced political violence with those who had not. The prevalence of alcohol misuse was not statistically different in the two groups (Eisenman 2003). A study of immigrant Bhutanese and native Nepalese torture survivors living in Nepal showed high levels of substance misuse in both groups (Emmelkamp 2002). Although many survivors of torture have strong religious convictions that reduce their likelihood of misusing substances, it is always important to enquire directly and assumptions should not be made on the basis of ethnic or religious background.

\section{The social effects of torture}

During an assessment much time will need to be dedicated to conducting a full social history. There is a paucity of research on the non-medical and non-psychological effects of torture, including the social, financial, occupational and relational consequences (McFarlane 2012). However, forced migration obviously has clear impacts on these and a thorough assessment should cover each of these areas. Torture can greatly affect an individual's family life. In the Bhutanese and Nepalese survivors of torture mentioned above, tobacco and alcohol use, relational problems, child abuse, social withdrawal and hopelessness were all common (Emmelkamp 2002). Family members of a torture survivor who have not been subjected to torture themselves can undergo 'secondary traumatisation’ (Jørgensen 2010). Secondary traumatisation is where individuals who live with 
a survivor of torture experience a threat to their own mental, physical or social functioning. A family history will prove to be very informative and may partly account for some of the symptoms experienced by the individual.

Limited research has looked at the impact of torture on functioning. A Danish study seeking to identify the main domains of functioning that may be impaired (Jørgensen 2010) found the following to be most important to an individual's functioning: problem-solving, carrying out daily routine, handling stress, conversation, using transportation, looking after their health, acquisition of goods and services, preparing meals, doing housework, assisting others, basic interpersonal interactions, formal relationships, informal social relationships, family relationships, school education, basic economic transactions and economic self-sufficiency. These were identified from a core set of symptoms from the World Health Organization's International Classification of Functioning, Disability and Health (ICF) (WHO 2008). This study demonstrated the pervasiveness of the functional impairment resulting from torture: impairment was seen in all the domains mentioned above. This highlights how holistic the assessment may need to be to fully describe any functional impairment in survivors of torture.

Current social circumstances are often overlooked in light of past trauma, but it is important to carefully consider the stability and suitability of an individual's present living arrangements, as these can often be the most significant current stressor.

\section{Interaction between different domains}

There are complex interactions between the multiple affected domains that must be considered when reviewing an individual. Consideration must be given to the process of migration and the current conditions that refugees find themselves in. Following the trauma of torture, the asylum process can be a secondary insult. Delay in the processing of an asylum application has a negative effect on mental health (Silove 1997). The vicious circle of the interaction between poor quality of life and poor mental health has been described among Ethiopian migrants (Araya 2007).

\section{Prognostic factors}

Female gender and older age have been shown to be risk factors for the development of PTSD in survivors of war and torture (Johnson 2008). Protective factors have also been described: these include religious belief and social and family supports. Preparedness for torture was shown to be protective against both psychopathology and PTSD symptoms (Basoglu 1997; Johnson 2008).

\section{Treatment of survivors of torture}

\section{General principles}

There is a paucity of research into the treatment and rehabilitation of survivors of torture (Jørgensen 2010). Research in this area is complicated by the heterogeneity of the populations, the varied forms of torture used, the diverse treatments and treatment settings and the range of training of the treatment providers. A meta-analysis of studies conducted with survivors of torture (McFarlane 2012) showed that $90 \%$ of the trials were of benefit to the participants; however, it acknowledged that publication bias was a problem in this area. Despite the limitations of this study it does give us some reason to be optimistic about the effect that treatment can have.

Jaranson \& Quiroga (2011) note that 'the context in which rehabilitation occurs affects the perception of torture's sequelae, diagnosis, treatment, and prognosis'. Treatment of survivors of torture can occur in four distinct settings (McFarlane 2012). First, survivors may remain in their homes in the country in which the torture was carried out. In this case many of the stressors will be ongoing and political instability may play a significant role in the patient's symptoms during treatment. Second, survivors may become 'displaced persons', displaced nationally (internally) or internationally (externally). Although these individuals have been forced to leave their homes, and often their friends and families, as 'displaced persons' they will not have applied for or been granted asylum. Third, survivors may become asylum seekers. The treatment of these individuals can be complicated by the uncertainty of their future. Fourth, survivors may become resettled refugees, granted asylum in another country.

The medical and psychological consequences of torture are often the focus of therapy in highincome countries. Although these consequences are present regardless of where individuals receive treatment, it is often the social and political consequences of torture that are more clearly identified by those working with survivors in their own countries (Jaranson 2011). The bias towards the medical model seen in high-income countries may have a significant impact on the clinician's understanding of symptoms and the treatment an individual receives. Box 4 lists various medical, psychosocial and educational interventions used in the rehabilitation of survivors for torture and Box 5 gives websites that provide helpful information. 
BOX 4 Rehabilitation of the survivors of torture

Medical intervention

- Medico-legal report

- Pharmacological treatment

Psychological therapy

- Cognitive-behavioural therapy

- Exposure therapy

- Testimonial therapy

- Narrative exposure therapy

- Psychodynamic psychotherapy

Social and alternative therapy and support

- Chinese massage

- Acupuncture

- Art therapy

- English language classes

- Computer skills classes

\section{Medical treatments}

Documentation of torture and medico-legal reports

The documentation of episodes of torture is a key initial step in the treatment of torture survivors and can serve many roles (Mandel 2007). For the survivor, it can aid advocacy and be used in legal and asylum proceedings. More generally, medico-legal reports can inform research, promote lobbying and help prevent torture.

The Istanbul Protocol (UN High Commissioner for Refugees 2004) gives guidance on writing medico-legal reports on torture and ill-treatment, but it is not widely used (Perera 2011). A full assessment in line with the Istanbul Protocol can take up to $7 \mathrm{~h}$. For many survivors of torture, the documentation of their experience is either absent or substandard. Lack of resources, the complex ethical considerations, possible re-traumatisation and the perceived divide between documentation

B0X 5 Useful resources

- International Rehabilitation Council for Torture Victims (IRCT): www.irct.org

- United Nations High Commissioner for Refugees (UNHCR): www.unhcr.org

- Red Cross international family tracing: www. redcross.org.uk/What-we-do/Finding-missing-family/ International-family-tracing

- The Istanbul Protocol: www.ohchr.org/Documents/ Publications/training8Rev1en.pdf and rehabilitation contribute to this problem. The importance of combining documentation and rehabilitation has been noted in the literature (Mandel 2007) but is yet to gain widespread acceptance in practice.

\section{Pharmacological treatments}

Much has been written on the pharmacological treatment of PTSD (Stein 2006; Bisson 2007), but few studies have addressed the response in survivors of torture. Two small, short-term, uncontrolled studies have been conducted in this population. The first (Kinzie 1989) assessed a combination of clonidine and imipramine in 9 Cambodian refugees. The second (Smajkic 2001) evaluated venlafaxine, sertraline or paroxetine in 32 Bosnian refugees. Both studies showed modest improvement in the symptoms of depression and PTSD, but were underpowered and had insufficient duration of follow up.

\section{Psychological treatments}

A wide range of psychological treatments have been used in populations who have experienced torture. However, evidence for these interventions is limited and is usually based on small studies (McFarlane 2012).

\section{Cognitive-behavioural therapy (CBT)}

Crumlish \& O'Rourke's systematic review of 10 RCTS of treatments for PTSD showed some limited evidence supporting the use of CBT in the rehabilitation of survivors of torture (Crumlish 2010). A number of high-quality RCTs of culturally adapted CBT have been carried out with resettled Cambodians. Improvements have been demonstrated in multiple outcomes, including PTSD symptoms, panic symptoms, systolic blood pressure, generalised anxiety and depression. One study also demonstrated improved quality of life with CBT, but the trial had significant methodological limitations, including the fact that the control group was receiving exposure therapy (Paunovic 2001).

\section{Exposure therapy}

Exposure therapy involves exposing patients to trauma-related memories within a therapeutic environment, with the aim of desensitising them to the stressful response. Little research has been done on exposure therapy alone for torture survivors, although its components are often included in other forms of therapy. One RCT comparing CBT with exposure therapy demonstrated that exposure therapy was as effective as CBT in reducing symptoms of anxiety, 
depression and PTSD up to 6-month follow-up (Paunovic 2001).

\section{Testimonial therapy}

This is a brief therapeutic intervention based on exposure therapy (Igreja 2004). It has been employed to treat individuals both in the environment they experienced their trauma and as refugees resettled in a high-income country (Weine 1998; Igreja 2004; Agger 2009). The therapist facilitates the traumatised individual's formation of a narrative of the experience of the trauma. This narrative includes a factual description of what happened, thoughts and emotions experienced by the individual and how this has affected their outlook on the future. The individual then delivers their story to their household. It is proposed that testimonial therapy works by allowing victims of trauma to ventilate feelings about their experience and to find meaning in the traumatic experience by reliving it. It is also felt that this therapy is a first step in challenging the social and political injustices that have resulted in torture or violence (Agger 2009).

Although some of the studies of testimonial therapy have shown an improvement in symptoms (Weine 1998), the only controlled study did not show a significant improvement compared with the control group (Igreja 2004). One of the uncontrolled studies looked at functioning as an outcome and showed significant improvement: this is of note as very few studies consider functioning as an outcome (Agger 2009).

\section{Narrative exposure therapy}

Narrative exposure therapy (NET) is a short-term intervention that blends components of testimonial therapy and CBT (Neuner 2004). In NET, the therapist and patient compile a record of the traumatic events experienced by repeatedly talking about the most traumatic of them. As this record is compiled, the therapist encourages the individual to re-experience the emotions of the events. The individual is asked about emotional, physiological, cognitive and behavioural experiencing that occur during the session. The session continues until the individual experiences habituation to the emotional response of the memories. At the end of therapy, the therapist gives the individual a written account of the trauma.

NET is thought to be effective for two reasons (Neuner 2004). First, it works as a form of exposure therapy. Second, victims of trauma often have a fragmented and distorted memory of the traumatic events (Ehlers 2000). The formation of a coherent narrative that occurs during NET may help lessen the disruption of autobiographical memory - autobiographical memory has been postulated to result in the sense of a severe and imminent threat. It has been suggested that this may reduce PTSD and anxiety symptoms.

The Crumlish \& O'Rourke review of PTSD treatments for refugees and asylum seekers demonstrated that no treatment had a solid evidence base, but NET showed the most promising results (Crumlish 2010). One well-conducted RCT of NET was undertaken with a population of asylum seekers in Germany (Neuner 2010). NET resulted in a statistically significant reduction in PTSD and somatic symptoms compared with standard community mental healthcare. Symptoms of depression were not significantly improved.

\section{Psychodynamic psychotherapy}

There has been minimal research into the effectiveness of psychodynamic therapy in treating survivors of torture and trauma. One uncontrolled study with significant methodological limitations showed some improvement in general psychiatric symptoms (Holmqvist 2006). Components of psychodynamic therapy are often included in other forms of therapy.

\section{Other therapeutic considerations}

\section{Alternative treatments}

In addition to psychological and pharmacological treatments there is a small but growing body of evidence for alternative treatments (Gray 2011). Expressive art therapies, acupuncture and Chinese massage are sometimes employed.

Many individuals may not be ready to engage in psychotherapy, especially if their asylum status is uncertain. For people in this situation, English language classes, informal support groups and computer skills training can prove invaluable and can greatly help them to integrate into their new environment. These measures would fit with the holistic treatment recommended by the IRCT (2014) for all torture survivors.

\section{The role of the interpreter}

Ideally, interpreters should be specially trained to work with survivors of torture. It is important to acknowledge the cultural background of both the survivor and the interpreter: although they may speak the same language, the interpreter may come from an ethnic group that the patient distrusts. Consideration must also be given to the gender of the interpreter: the patient may have a preference, especially if they want to discuss sexual assaults or rape. 


\section{Case vignette}

The following fictitious vignette illustrates a typical clinical presentation.

A 26-year-old Iraqi woman was referred to her local community mental health team by her GP, who thought that she was depressed. In Iraq she had been imprisoned without trial and during this time had been subjected to beatings and sexual assault because of her family's political views. She also witnessed the murder of family members and the torture of other people. She had escaped Iraq with the help of a family member and had made her way to Ireland. She spoke no English. On arrival she applied for asylum.

Assessment was delayed twice: first, because a male interpreter was provided and she was uncomfortable recounting her story in the presence of a man; then because, during the second interview, she became very concerned that the female interpreter was working for the Iraqi government. A third interpreter was found and a full assessment was carried out.

The woman's main reported problems were insomnia and chronic abdominal pain. She denied having low mood, but further questioning revealed reduced energy, anhedonia, reduced appetite, insomnia and intense guilt about having left her family. Her insomnia was partly explained by nightmares she was experiencing. She also experienced flashbacks and hyperarousal. No features of psychosis were present and the potential paranoia shown with the second interpreter was not delusional: she was able to give clear justification for her concerns. Investigations had been organised for her abdominal pain, but she had not attended them. She met diagnostic criteria for moderate depression and PTSD.

She was started on fluoxetine and referred to a local service providing psychological interventions to asylum seekers. Extensive work was done liaising with the GP to ensure full investigation of her abdominal symptoms. Initially, she disengaged from the psychological treatments, but continued to attend a support group and English language classes.

Her initial asylum application was rejected and following this she presented to a hospital emergency department after an episode of self-poisoning. She had a brief admission and was discharged to the community mental health team. After a lengthy appeal she was granted refugee status. Shortly after this she re-engaged with the psychological interventions. Her symptoms improved significantly over the following 6 months and she was discharged to the care of her GP.

This vignette demonstrates many key components of the assessment and treatment of survivors of torture. There is a great need for cultural awareness and sensitivity. Caution needs to be exercised when potentially delusional symptoms are elicited, as some may be justified or culturally based. The individual's social context, especially if they are seeking asylum, has great impact. Often, the uncertainty and stress of going through the asylum process makes psychological interventions difficult. A mental health team may need to help the individual to access medical services as mental health problems, language, cultural differences and fear of authorities may act as barriers to receiving medical care.

\section{Conclusions}

The UNHCR is currently concerned about more than 35 million refugees (UNHCR 2014). Many of these forced migrants have experienced torture and political violence. The physical, psychological and social effects of torture are wide-reaching and long-standing. Anxiety, PTSD and depression are all greatly increased in survivors of torture. Many additional considerations must be kept in mind when assessing survivors. These include: the asylum process, interview style and cultural differences. Although the evidence base remains limited, the effects of torture have been shown to improve with treatment. For example, CBT and NET are two of the best-evidenced treatments to date for PTSD and anxiety symptoms. The treatment and rehabilitation of torture survivors is a growing area of interest for many researchers and it is to be hoped that other effective interventions will soon be identified to help this growing population of innocent victims.

\section{References}

Agger I, Raghuvanshi L, Shabana Khan S, et al (2009) Testimonial therapy: a pilot project to improve psychological wellbeing among survivors of torture in India. Torture, 19: 204-17.

Amnesty International (2010) Amnesty International Report 2010: The State of the World's Human Rights. Amnesty International.

Araya M, Chotai J, Komproe IH, et al (2007) Effect of trauma on quality of life as mediated by mental distress and moderated by coping and social support among postconflict displaced Ethiopians. Quality of Life Research, 16: 915-27.

Basoglu M, Mineka S, Paker M, et al (1997) Psychological preparedness for trauma as a protective factor in survivors of torture. Psychological Medicine, 27: 1421-33.

Bisson Jl (2007) Pharmacological treatment of post-traumatic stress disorder. Advances in Psychiatric Treatment, 13: 119-26.

Carlsson JM, Olsen DR, Mortensen EL, et al (2006) Mental health and health-related quality of life: a 10-year follow-up of tortured refugees. Journal of Nervous and Mental Disease, 194: 725-31.

Cloitre M, Garvert DW, Brewin CR, et al (2013) Evidence for proposed ICD-11 PTSD and complex PTSD: a latent profile analysis. European Journal of Psychotraumatology, 4: doi: 10.3402/ejpt.v4i0.20706.

Crumlish N, O'Rourke K (2010) A systematic review of treatments for post-traumatic stress disorder among refugees and asylum seekers. Journal of Nervous and Mental Disease, 198: 237-51.

Curtice M (2010) The European Convention on Human Rights: an update on Article 3 case law. Advances in Psychiatric Treatment, 16: 199-206.

Ehlers A, Clark DM (2000) A cognitive model of posttraumatic stress disorder. Behavioral Research and Therapy, 38: 319-45. 


\section{MCO answers \\ 1 c 2 e $3 d \quad 4$ a $5 d$}

Eisenman DP, Gelberg L, Liu H, et al (2003) Mental health and healthrelated quality of life among adult Latino primary care patients living in the United States with previous exposure to political violence. JAMA, 290: 627-34

Elklit A, Hyland P, Shevlin M (2014) Evidence of symptom profiles consistent with posttraumatic stress disorder and complex posttraumatic stress disorder in different trauma samples. European Journal of Psychotraumatology, 5: doi 10.3402/ejpt.v5.24221.

Emmelkamp J, Komproe IH, Van Ommeren M, et al (2002) The relation between coping, social support and psychological and somatic symptoms among torture survivors in Nepal. Psychological Medicine, 32: $1465-70$

Fazel M, Wheeler J, Danesh J (2005) Prevalence of serious mental disorder in 7000 refugees resettled in western countries: a systematic review. Lancet, 365: 1309-14.

Gray AEL (2011) Expressive arts therapies: working with survivors of torture. Torture, 21: 39-47.

Holmqvist R, Andersen K, Anjum T, et al (2006) Change in self-image and PTSD symptoms in short-term therapies with traumatized refugees. Psychoanalytic Psychotherapy, 20: 251-65.

Igreja V, Kleijn WC, Schreuder BJN, et al (2004) Testimony method to ameliorate post-traumatic stress symptoms: community-based intervention study with Mozambican civil war survivors. British Journal of Psychiatry, 184: 251-7.

International Rehabilitation Council for Torture Victims (2010) The IRCT Strategy Framework 2010-2014. IRCT Communications.

International Rehabilitation Council for Torture Victims (2012) Global Report on the UN International Day in Support of Victims of Torture. IRCT Communications.

International Rehabilitation Council for Torture Victims (2014) The Copenhagen Declaration on Right to Rehabilitation. IRCT Communications.

Jakobsen M, Sveaass N, Eide Johansen L, et al (2007) Psykisk helse i mottak: utprøving av instrumenter til kartlegging av psykisk helse hos nyankomne asylsøkere (Rapport nr. 4/2007) [Mental Health in Reception Centres: Testing Instruments for Identifying Mental Health of Asylum Seekers] (Report No. 4/2007). Norwegian Centre for Violence and Traumatic Stress Studies, University of Oslo.

Jaranson JM, Quiroga J (2011) Evaluating the services of torture rehabilitation programmes: history and recommendations. Torture, 21: $98-140$

Johnson H, Thompson A (2008) The development and maintenance of post-traumatic stress disorder (PTSD) in civilian adult survivors of war trauma and torture: a review. Clinical Psychology Review, 28: 36-47.

Jørgensen U, Melchiorsen H, Gottlieb AG, et al (2010) Using the International Classification of Functioning, Disability and Health (ICF) to describe the functioning of traumatised refugees. Torture, 20: 57-75.

Knefel M, Lueger-Schuster B (2013) An evaluation of ICD-11 PTSD and complex PTSD criteria in a sample of adult survivors of childhood institutional abuse. European Journal of Psychotraumatology, 4: doi: 10.3402/ejpt.v4i0.22608.

Kinzie JD, Leung P (1989) Clonidine in Cambodian patients with posttraumatic stress disorder. Journal of Nervous and Mental Disease, 177: $546-50$

Lanius RA, Brand B, Vermetten E, et al (2012) The dissociative subtype of posttraumatic stress disorder: rationale, clinical and neurobiological evidence, and implications. Depression and Anxiety, 29: 701-8.

Mandel L, Worm L (2007) Documentation of torture victims: implementation of medico-legal protocols. Torture, 17: 18-26.

Masmas TN, Møller E, Buhmannr C, et al (2008) Asylum seekers in Denmark: a study of health status and grade of traumatization of newly arrived asylum seekers. Torture, 18: 77-86.

McDonnell M, Robjant K, Katona C (2013) Complex posttraumatic stress disorder and survivors of human rights violations. Current Opinion in Psychiatry, 26: 1-6.
McFarlane CA, Kaplan I (2012) Evidence-based psychological interventions for adult survivors of torture and trauma: a 30-year review. Transcultural Psychiatry, 49: 539-67.

Montgomery E, Foldspang A (1994) Criterion-related validity of screening for exposure to torture. Danish Medical Bulletin, 41: 588-91.

Moreno A, Grodin MA (2002) Torture and its neurological sequelae. Spinal Cord, 40: 213-23.

Neuner F, Kurreck S, Ruf M, et al (2010) Can asylum-seekers with posttraumatic stress disorder be successfully treated? A randomized controlled pilot study. Cognitive Behaviour Therapy, 39: 81-91.

Neuner F, Schauer M, Klaschik C, et al (2004) A comparison of narrative exposure therapy, supportive counseling, and psychoeducation for treating posttraumatic stress disorder in African refugee settlement. Journal of Consulting and Clinical Psychology, 72: 579-87.

Olsen DR, Montgomery E, Bøjholm S, et al (2007) Prevalence of pain in the head, back and feet in refugees previously exposed to torture: a tenyear follow-up study. Disability and Rehabilitation, 29: 163-71.

Paunovic N, Ost L (2001) Cognitive-behavior therapy vs exposure therapy in the treatment of PTSD in refugees. Behaviour Research and Therapy, 39: 1183-97.

Perera C, Verghese A (2011) Implementation of Istanbul Protocol for effective documentation of torture: review of Sri Lankan perspectives. Journal of Forensic and Legal Medicine, 18: 1-5.

Resick PA, Bovin MJ, Calloway AL, et al (2012) A critical evaluation of the complex PTSD literature: implications for DSM-5. Journal of Traumatic Stress, 25: 241-51.

Robertson CL, Halcon L, Savik K, et al (2006) Somali and Oromo refugee women: trauma and associated factors. Journal of Advanced Nursing, 56: $577-87$.

Shrestha NM, Sharma B, Van Ommeren M, et al (1998) Impact of torture on refugees displaced within the developing world: symptomatology among Bhutanese refugees in Nepal. JAMA, 280: 443-8.

Silove D, Sinnerbrink I, Field A, et al (1997) Anxiety, depression and PTSD in asylum-seekers: associations with pre-migration trauma and postmigration stressors. British Journal of Psychiatry, 170: 351-7.

Smajkic A, Weine S, Djuric-Bijedic Z, et al (2001) Sertraline, paroxetine, and venlafaxine in refugee posttraumatic stress disorder with depression symptoms. Journal of Traumatic Stress, 14: 445-52.

Steel Z, Chey T, Silove D, et al (2009) Association of torture and other potentially traumatic events with mental health outcomes among populations exposed to mass conflict and displacement: a systematic review and meta-analysis. JAMA, 302: 537-49

Stein D, Isper J, Seedat S (2006) Pharmacotherapy for post traumatic stress disorder (PTSD). Cochrane Database of Systematic Reviews, 1 . CD002795

United Nations (1984) Convention against Torture and Other Cruel, Inhuman or Degrading Treatment or Punishment (resolution 39/46). United Nations.

UN High Commissioner for Refugees (2004) Istanbul Protocol - Manual on the Effective Investigation and Documentation of Torture and Other Cruel, Inhuman or Degrading Treatment and Punishment. UNHCR.

UN High Commissioner for Refugees (2014) Thank you for supporting refugees in 2014. UNHCR (http://www.unhcr.org.uk/news-and-views/ news-list/news-detail/article/thank-you-for-supporting-refugeesin-2014.html). Accessed 12 Jan 2015

van Ommeren M, Komproe I, Cardeña E, et al (2004) Mental illness among Bhutanese shamans in Nepal. Journal of Nervous and Mental Disease, 192: 313-7.

Weine SM, Kulenovic AD, Pavkovic I, et al (1998) Testimony psychotherapy in Bosnian refugees: a pilot study. American Journal of Psychiatry, 155 $1720-6$.

Wenzel T (2007) Torture. Current Opinion in Psychiatry, 20: 491-6.

World Health Organization (2008) International Classification of Functioning, Disability and Health (ICF). WHO. 
MCQs

Select the single best option for each question stem

1 Regarding torture in the modern world:

a according to Amnesty International, $50 \%$ of the $\mathrm{G}-20$ countries were practising torture or otherwise ill-treating individuals in 2010

b torture is a well-defined concept in the medical literature

c torture is still thought to be practised in over 130 countries

$d$ the problem of torture is limited to low- and medium-income countries

e torture must involve physical pain to meet the UN's definition.

\section{Regarding the physical consequences of} torture:

a hypertension is an example of the direct consequences of torture

b symptoms of frontal lobe damage are easily identified in people who have experienced torture

c the physical consequences are similar throughout the world d the physical consequences are much less important than the psychological sequelae

e the physical consequences can seriously impair social functioning.

3 Regarding psychological sequelae of torture:

a anxiety disorders are twice as common in displaced survivors of torture than in displaced people who have not experienced torture

b the psychological symptoms are generally short lived

c in $25 \%$ of patients, psychological symptoms do not improve despite interventions

d depression is $50 \%$ more common in displaced survivors of torture than in displaced people who have not experienced torture

e PTSD is five times more common in displaced survivors of torture than in displaced people who have not experienced torture.

4 Regarding the medical assessment of survivors of torture:

a medico-legal reports can aid advocacy and can be used in legal and asylum proceedings b medico-legal reports result in the retraumatising of survivors of torture

c assessments are usually brief, taking no more than $1 \mathrm{~h}$

d the Budapest Protocol provides guidance on writing medico-legal reports for survivors of torture

e male interpreters should never be used.

5 Regarding the psychological treatment of survivors of torture:

a there have been many large high-quality studies

b there is a solid evidence base for psychodynamic psychotherapy

c there is no evidence base for CBT

d narrative exposure therapy has some limited evidence base

e exposure therapy concludes with the individual attending therapy being presented with a written account of their experiences. 